\title{
CONSERVATIVE AVERAGING METHOD FOR SOLVING SOME NONLINEAR HEAT TRANSFER PROBLEMS RELATED TO COMBUSTION
}

\author{
Aivars Aboltins ${ }^{1}$, Ilmars Kangro ${ }^{2}$, Harijs Kalis ${ }^{3}$ \\ ${ }^{1}$ Latvia University of Life Sciences and Technologies, Latvia; ${ }^{2}$ Rezekne Academy of Technologies, \\ Latvia; ${ }^{3}$ Institute of Mathematics and Computer Science, University of Latvia, Latvia \\ aivars.aboltins@1lu.lv, ilmars.kangro@rta.lv, harijs.kalis@lu.lv
}

\begin{abstract}
In the paper we consider some nonlinear heat transfer problems related to combustion. At first, the coincidence of the exact solution of the studied boundary value problem with the approximate solution - the designed exponential type spline function is proved. The second problem under question is the combustion process with Arrhenius kinetics using single step chemical reactions. The exothermic chemical reactions are modelled by single step of fuel and oxidant, at the inlet the constant axial velocity is given. Numerical solution with Matlab routines "pdepe" and exponential type spline function are obtained. Some numerical and experimental results are given. As a practical example of solving a nonlinear system of the parabolic type of PDE the calculation of temperature and moisture in the wood sample in its burning process is considered. The profiles of temperature and moisture depending on time in the different places of wood sample are obtained.
\end{abstract}

Keywords: conservative averaging method; diffusion-convection boundary value problem; exothermic chemical reaction; exponential type splines, wood-burning.

\section{Introduction}

The solutions of 1D diffusion and diffusion-convection boundary value problems (BVP) for partial differential equations (PDE) are applicable to a number of practical problems / tasks related to mathematical modelling of combustion processes [1-3]. For example, the authors of the present article have studied transfer problems involving combustion of gypsum board products in previous publications - the heat transfer problem for two layered gypsum board products exposed to fire [4], the heat and moisture distribution problem in the drying process for porous two layered gypsum board products [5]. In recent years, based on a general approach to modelling flame through chemical reactions [6; 7], the problems/tasks of mathematical modelling have been significantly supplemented by modelling biomass combustion processes with reaction diffusion equations based on gas dynamics components, temperature, chemical response, fuel concentrations and electric field calculations using Matlab software mathematical and numerical support [8;9].

However, in the event of a BVP solution, additional difficulties are often encountered in defining the conditions of continuity by modelling layered environmental (multi-layer environment) objects with the Matlab "pdepe" programme, and therefore, the resulting numerical solution does not describe the process sufficiently (correctly). This occurs, for example, by solving the diffusion-convection BVP of PDE in a multi-layer environment with different values of convection coefficients (different convection velocity) of each layer. Therefore, this publication also continues the themes that have been launched in the past: obtaining a solution to the boundary value problems under question in the form of analytical formulas, based on the conservative averaging method (CAM), namely, the development of the approximate solution to the BVP in the form of integral exponential or hyperbolic type splines. A study has been carried out in this publication on the appropriateness of the exact solution of the studied boundary value problem to the exponential type spline function newly created, and it has been demonstrated that they completely coincide in a linear case.

It allowed the use of the created integral exponential spline function to solve BVP viewed in a given publication alongside the used Matlab software. As a result, in addition to the traditional math methods used by other authors $[9 ; 10]$ (finite-difference methods, alternating direction method), new engineering solutions were obtained for the problems / tasks under consideration with the created conservative averaging method, taking into account its advantages in theoretical meaning and in terms of applying math software, that is, in the performance of numerical calculations.

\section{The mathematical model of diffusion-convection boundary-value problem}

We are studying diffusion-convection processes in multi-layer environments (layered environments), where different convection speeds are observed in vertical direction. For this purpose the adequate mathematical model created is suitable for modelling and investigating the following 
physical processes: the calculation of temperature and humidity concentrations in heating, drying or moistening processes of wood or other multi-layer material; filtration problems, where a contaminated solution, which is flowing through a layered material (sorbent), is being gradually purged.

Therefore, in this chapter we consider the diffusion-convection boundary-value problem (BVP) in multi-layered plane domain in z-direction with different convection velocity in every layer (1-5). In this case, the solution to the BVP is discontinuous and can easily be gained by solving this BVP with the conservative averaging method (CAM), using the exponential type spline function.

The 1-D domain $\Lambda=\{z \mid 0 \leq z \leq \mathrm{L}\}$ contains $N$ layers $\Lambda_{i}=\left\{z \mid z \in\left(z_{i-1}, z_{i}\right)\right\}, i=\overline{1, N}, z_{0}=0, z_{N}=L$. We consider the following partial differential equation (PDE) (1) with continuous conditions (CCs) (4-5) and boundary conditions (BCs) (2-3):

$$
\left\{\begin{array}{l}
D_{i} \frac{\partial^{2}}{\partial z^{2}} u_{i}(z)+r_{i} \frac{\partial u_{i}(z)}{\partial z}+F_{i}=0, z \in\left(z_{i-1}, z_{i}\right), i=\overline{1, N}, \\
D_{1} \frac{\partial u_{1}(0)}{\partial z}-\alpha\left(u_{1}(0)-u_{0}\right)=0, z=0, \\
D_{N} \frac{\partial u_{N}(L)}{\partial z}+\beta\left(u_{N}(L)-u_{L}\right)=0, z=L, \\
D_{i} \frac{\partial u_{i}\left(z_{i}\right)}{\partial z}=D_{i+1} \frac{\partial u_{i+1}\left(z_{i}\right)}{\partial z}, i=\overline{1, N-1,} \\
r_{i} u_{i}\left(z_{i}\right)=r_{i+1} u_{i+1}\left(z_{i}\right),
\end{array}\right.
$$

where $u_{i}=u_{i}(z)-$ concentration function in every layer;

$D_{i}-$ constant diffusion coefficients;

$\alpha, \beta$ - constant mass transfer coefficients in the 3-rd type BCs;

$u_{0}, u_{L}-$ given concentration on the boundary;

$r_{i}-$ constant convection velocity in very layer;

$F_{i}-$ constant source terms.

It is possible to obtain an analytical solution for the BVP (1-5) in the following form:

$$
u_{a i}(z)=m_{i}\left(z-z_{i}^{*}\right)+e_{i} \exp \left(a_{i 1}\left(z-z_{i}^{*}\right)\right)+C_{i}, a_{i 1}=-r_{i} / D_{i}, m_{i}=-F_{i} / r_{i}, z_{i}^{*}=\left(z_{i-1}+z_{i}\right) / 2
$$

Applying BCs (2-3) and CCs (4-5), it is possible to obtain 2N-linear algebraic equations for determining the unknown coefficients $e_{i}$ and then $C_{i}$.

According to the conservative averaging method (CAM) [11], we use the integral exponential type splines:

$$
u_{i}(z)=u_{i z}+m_{i z}\left(z-z_{i}^{*}\right)+e_{i z}\left(\exp \left(a_{i 1}\left(z-z_{i}^{*}\right)-q_{i}\right), i=\overline{1, N},\right.
$$

where

$$
\begin{aligned}
& u_{i z}=\left(H_{i}\right)^{-1} \int_{z_{i-1}}^{z_{i}} u_{i}(z) d z, \int_{z_{i-1}}^{z_{i}}\left(z-z_{i}^{*}\right) d z=\int_{z_{i-1}}^{z_{i}}\left(\exp \left(a_{i 1}\left(z-z_{i}^{*}\right)-q_{i}\right)\right) d z=0, \\
& m_{i z}=-F_{i} / r_{i}, z_{i}^{*}=\left(z_{i-1}+z_{i}\right) / 2, z \in\left[z_{i-1}, z_{i}\right], q_{i}=\frac{2}{a_{i 1} H_{i}} \sinh \frac{a_{i 1} H_{i}}{2} .
\end{aligned}
$$

From BCs (2-3) at $z=0, z=L$ and the CCs (4-5) we can determine the unknown coefficients $e_{i z}$ and $m_{i z}$ depending of the mean integral values $u_{i z}$.

Using the average integral values of the differential equation, that is, by dividing by $D_{i}$, by integrating the equation (1) by a variable $z$ within each layer and dividing it by each layer's height $H_{i}$, then applying the spline function (7), we get the following identity:

$$
2 e_{i z} a_{i 1} \sinh \left(0.5 a_{i 1} H_{i}\right) / H_{i}-a_{i 1}\left(m_{i z} H_{i}+2 e_{i z} \sinh \left(0.5 a_{i 1} H_{i}\right)\right) / H_{i}+F_{i} / D_{i}=0, i=\overline{1, N} \text {. }
$$


The mean integral values $u_{i z}$ follow from the identity obtained: $u_{i z}=C_{i}+e_{i z} q_{i}$ or $C_{i}=u_{i z}-e_{i z} q_{i}$. We can see that by inserting the found $C_{i}, e_{i}=e_{i z}, m_{i}=e_{i z}$ in the analytical solution (6) formula (7) is obtained, that is, the created exponential type splines entirely coincide with the exact solution for the BVP studied.

\subsection{Numerical results for diffusion-convection BVP (1-5) in two layers}

The numeric experiment for solving the BVP (1-5) was conducted with Matlab at the following parameter values:

- $\quad N=L=2, H_{1}=H_{2}=1, F_{1}=1, F_{2}=10, \alpha=100, \beta=1, u_{0}=0, u_{L}=1$;

- at $r_{1}=1, r_{2}=2, D_{1}=D_{2}=0.01$, we have discontinuous solutions with $u_{1}(1)=12.60$, $u_{1}(1)=6.30$ and with averaged values $u_{1 z}=11.75, u_{2 z}=3.99$ (see Fig. 1 ),

- at at $r_{1}=r_{2}=2, D_{1}=0.01, D_{2}=0.02$, we have continuous solutions and discontinuous derivatives with $u_{1}(1)=u_{2}(1)=6.525$ and following averaged $u_{1 z}=6.43, u_{2 z}=4.45$ (see Fig. 2).

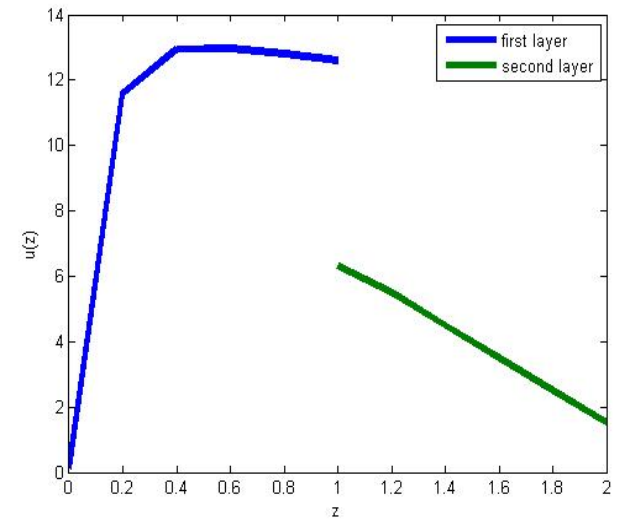

Fig. 1. Solutions for $r_{1}=1, r_{2}=2, D_{1}=D_{2}=0.01$

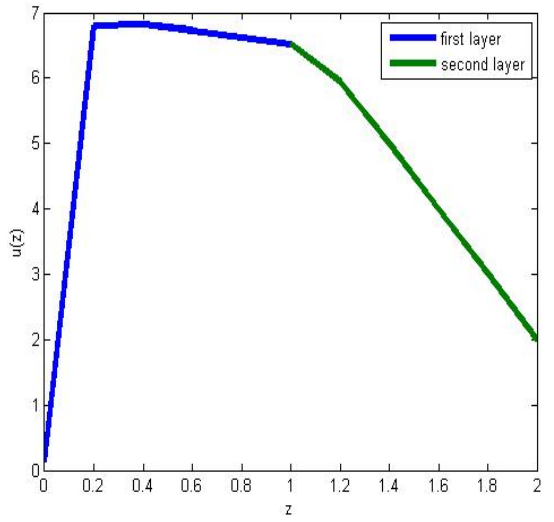

Fig. 2. Solutions for $r_{1}=r_{2}=2, D_{1}=0.01$, $D_{2}=0.02$

\section{The mathematical model of the combustion processes with reaction-diffusion equations}

Literature sources $[12 ; 13]$ deal with the following studied practical problem - the impact of the electric field on the combustion processes descriptors.

The mathematical model created describes the electric field influence on the combustion characteristics, using the approximation of $2 \mathrm{D}$ axially symmetric compressible swirling flow and chemical reactions with account of the development of $A \Rightarrow B \Leftrightarrow C$ kinetics ( $A$ - reactant, $B$ - intermediate product, $\mathrm{C}$ - final product) downstream the cylindrical combustion chamber

The exothermic chemical reaction [10] under question has been modelled by single step of fuel $(F)$ and oxidant $\left(O_{2}\right)$ combining to produce products $(P)$ and heat $(H)$. The generic kinetic schemes of models with one-step chemistry are: $F \Rightarrow P+H$, where the temperature dependent rate of the reaction is given by one-step Arrhenius kinetics of the 1st order $-K(T)=\exp \left(-T_{\alpha} / T_{0}\right), T_{\alpha}$ is the activation temperature. The approximated numerical solving of nonlinear problems in combustion processes as usually is based and has been conducted on implicit finite-difference methods and alternating direction (ADI) methods, reflected in $[10 ; 12]$.

The combustion process in the present article is studied in the $z$-plane $\left(0<z<z_{0}=0.1, \mathrm{~m}\right)$ with temperature $T=T(z, t), \mathrm{K}$ and simple exothermic chemical reaction by first-order Arrhenius kinetics with mass fraction $C=C(z, t)$, mol of the reactant in the time $t$ is modelled.

The thermo-physical parameters of the heat and reaction are assumed constant: $D=5 \cdot 10^{-5} \mathrm{~m}^{2} \cdot \mathrm{s}^{-1}$ is the molecular diffusivity, $\lambda=5 \cdot 10^{-5} \mathrm{~J} \cdot \mathrm{s}^{-1} \cdot(\mathrm{m} \cdot \mathrm{K})^{-1}$ - the thermal conductivity, $c_{p}=1000 \mathrm{~J} \cdot(\mathrm{kg} \cdot \mathrm{K})^{-1}-$ the specific heat at constant pressure, $B=1.5 \cdot 10^{6} \mathrm{~J} \cdot \mathrm{kg}^{-1}, A^{\prime}=10^{4} \mathrm{~s}^{-1}, E=2.5 \cdot 10^{4} \mathrm{~J} \cdot \mathrm{mol}^{-1}$ are the specific heat release, the reaction-rate pre-exponential factor and the activation energy, $R-$ the universal gas constant. Let $T_{0}=300 \mathrm{~K}, \rho_{0}=1 \mathrm{~kg} \cdot \mathrm{m}^{-3}, C=C_{0}=1 \mathrm{~mol}-$ the initial temperature, nominal density, mass fraction for concentration of fuel and axial velocity with uniform stream $U_{0}=0.01 \mathrm{~m} \cdot \mathrm{s}^{-1}$ at the inlet $z=0$. 
The equations were put in the dimensionless form scaling all the lengths to $z_{0}$, the density to $\rho_{0}$, the velocities $u_{z}$ to $U_{0}$, the temperature to $T_{0}$, the specific heat release $B$ to $c_{p} / T_{0}$, the reaction-rate preexponential factor $A^{\prime}$ to $U_{0} / z_{0}, A=A^{\prime} /\left(z_{0} U_{0}\right)$, the activation energy $E$ to $R / T_{0}$.

The following parameters are used:

- $\quad P_{e}=\left(z_{0} U_{0}\right) / D, L_{e}=\lambda /\left(c_{p} D \rho_{0}\right)-$ Peclet and Lewis numbers;

- $P_{1}=L_{e} / P_{e}, P_{2}=P_{e}^{-1}, \beta=B /\left(c_{p} / T_{0}\right), \delta=E /\left(R T_{0}\right)$ - the scaled heat-release and activationenergy.

For the dimensionless parameters $t, x=z / z_{0}, w=u_{z} / u_{0}$ we have the following 2 reactionsdiffusions equations:

$$
\left\{\begin{array}{l}
\frac{\partial T}{\partial t}+w \frac{\partial T}{\partial x}=\frac{P_{1}}{\rho} \frac{\partial^{2} T}{\partial x^{2}}+\beta A C \exp \left(-\frac{\delta}{T}\right) \\
\frac{\partial C}{\partial t}+w \frac{\partial C}{\partial x}=P_{2} \frac{\partial^{2} C}{\partial x^{2}}-A C \exp \left(-\frac{\delta}{T}\right)
\end{array}\right.
$$

We use the following boundary conditions:

1. at the outlet $x=x_{0}-\partial s / \partial x=0, s=T, \mathrm{C}$;

2. at the inlet $x=0, T=1, C(x, 0)=\exp (-\alpha x), \alpha \in[0,6]-$ is the parameter for the initial fuel amount in the plate.

This approach seeks obtaining the stationary solution as the asymptotic limit of the solutions of the nonstationary equations in time.

\subsection{Some numerical results with reaction-diffusion equations for combustion processes}

The influence of the molecular diffusivity and thermal conductivity on the main characteristics of the undisturbed flame flow is obtained for $\beta=5$.

Such results have been obtained - that at the constant molecular diffusivity $D$ the decreasing of the thermal conductivity $\lambda\left(P_{1}=0.01, L_{e}=0.1\right)$ leads to an increasing of the reaction rate and maximal values of the temperature, but at the constant thermal conductivity $\lambda$ the decreasing of the molecular diffusivity $D\left(P_{2}=0.01, L_{e}=10\right)$ results in an increasing of the maximal density and in a decreasing of the temperature and reaction rate.

For fixed values of velocities $w=1$ and $P_{1}=P_{2}=0.1$ the heat-reaction problem has been solved numerically, using finite differences approximation and due to integral exponential type spline function for two cases: $\rho=1 / T$ (small Mach numbers for compressible fluid) and $\rho=1$ (incompressible flow). If $\rho=1 / T$, then we have for maximal and averaged values of temperature: $T_{\max }=2.957$ (Fig. 3) and for $\rho=1$ we have $T_{\max }=5.969$ (Fig. 4).

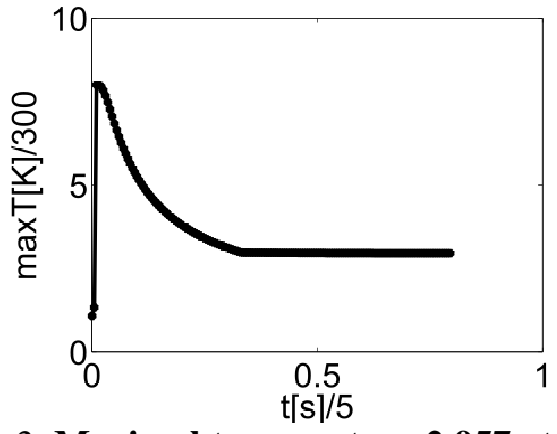

Fig. 3. Maximal temperature 2.957 at outlet depending on time $t$ at $\rho=1 / T$

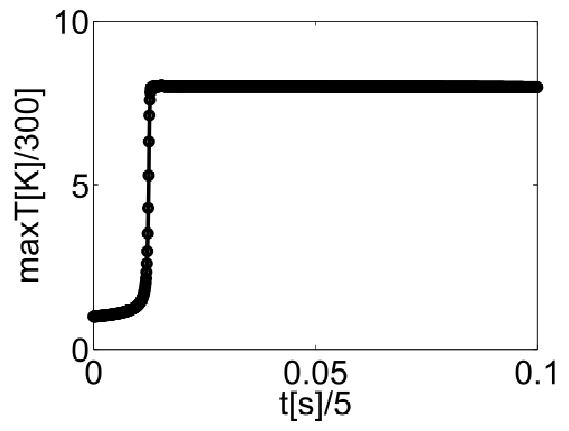

Fig. 4. Maximal temperature 5.969 depending on time $t$ at $\rho=1$

We have solved the 1D reaction-diffusion problem (8) $(\rho=1)$, where $A=5 \cdot 10^{4}, \beta=5, \delta=10$, $t_{f}=1, x_{0}=2, \alpha=6$.

Depending on the values of the parameter $w$, the following temperatures are obtained: $w=1\left(T_{\max }=6.0881, T\left(x_{0}, t_{f}\right)=T(2,1)=1.295\right)$ and $w=4\left(T_{\max }=6.114, T\left(x_{0}, t_{f}\right)=T(2,1)=1.248\right)$, we have profiles of temperature depending on $x$ in Fig. 5, Fig. 6. 


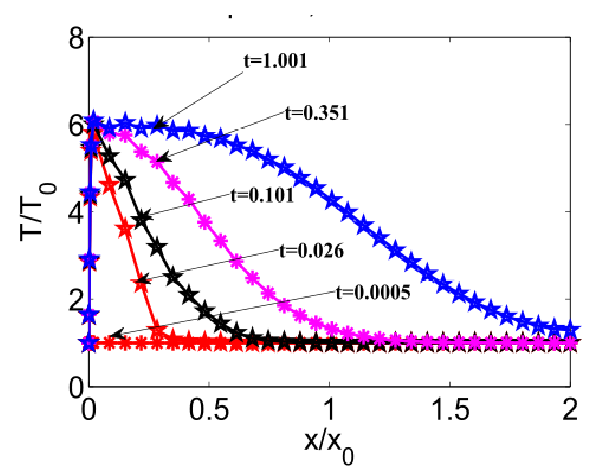

Fig. 5. Profile of temperature depending on $x$ in fixed time $t$ at $w=1, \alpha=6, P_{1}=P_{2}=0.1$, $T_{\max }=6.008$

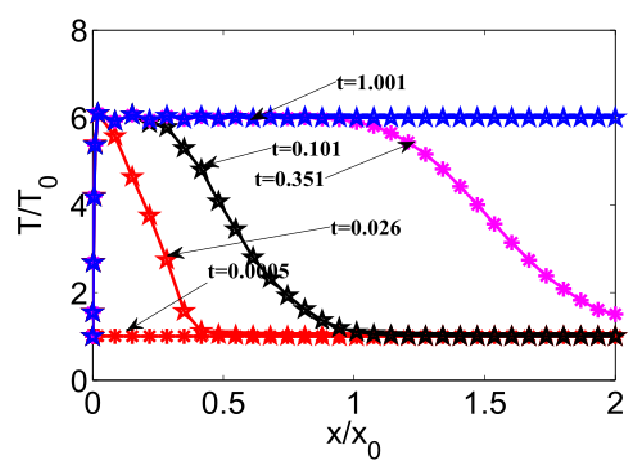

Fig. 6. Profile of temperature depending on $x$ in fixed time $t$ at $w=4, \alpha=6, P_{1}=P_{2}=0.1$, $T_{\max }=6.114$

The higher burning temperature is achieved in the case of higher axial velocity $w$, as seen at the end time $t=1.001$ ( $w=4$, Fig.6), the temperature is no longer decreasing, but remains constant throughout the $x$ change interval, i.e. the flame spreads through the whole combustion chamber.

\section{Mathematical modelling of the wood-burning process}

We are studying the spread of temperature and moisture in the wood sample in its burning process $[14 ; 15]$. According to the physical process to be studied (Fig. 7) mathematical model (9) - a nonlinear system of the parabolic type of PDE has been developed.

The unknown functions $T=T(x, t)$ (temperature) and $u=u(x, t)$ (moisture) depend on the time $t$ and horizontal coordinates $x$, taking into account fluctuations in the heat flow and the heat source term that characterizes the heat spreading in the wood burning furnace.

$$
\left\{\begin{array}{l}
\frac{\partial T}{\partial t}=\frac{\partial}{\partial x}\left(a(T) \frac{\partial T}{\partial x}\right)-\frac{q}{c_{p} \rho} \frac{\partial u}{\partial t}+\frac{\sigma \varepsilon S}{c_{p} \rho} T^{4} \\
\frac{\partial u}{\partial t}=\alpha \frac{\partial u}{\partial x}
\end{array}\right.
$$

where $t \in\left[0, t_{f}\right]-$ time in hours, $t_{f}=3 \mathrm{~h}$;

$x \in[0, L]-$ thickness of the sample, $L=5 \mathrm{dm}$;

$T$ - temperature, $\mathrm{K}$;

$u$ - moisture content, \%;

$k(T) \in[0.15,0.20]-$ thermal conductivity decreasing on $T, \mathrm{~W} \cdot(\mathrm{m} \cdot \mathrm{K})^{-1}$, in Fig. 8 the thermal conductivity $k(T)$ is represented depending on the temperature $T \in[0,1000],{ }^{\circ} \mathrm{C}$ using spline approximation;

$k_{0}=0.15 \mathrm{~W} \cdot(\mathrm{m} \cdot \mathrm{K})^{-1}-$ the thermal conductivity in the combustor by $x=L$;

$c_{p}=2390 \mathrm{~J} \cdot(\mathrm{kg} \cdot \mathrm{K})^{-1}-$ specific heat at a constant pressure $\rho=\rho_{0}(1+u / 100)$;

$\rho_{0}=700 \mathrm{~kg} \cdot \mathrm{m}^{-3}-$ density of the wood;

$q=2256000 \mathrm{~J} \cdot \mathrm{m}^{-3}-$ the heat amount for evaporating of water;

$\sigma=5.67 \cdot 10^{-8} \mathrm{~J} \cdot \mathrm{s}^{-1} \cdot \mathrm{m}^{-2} \cdot \mathrm{K}^{-4}-$ Stefan-Boltzmann constant;

$\varepsilon=0.95$ - surface of emissivity;

$S=100 \mathrm{~cm}^{2}-$ surface of radiation;

$F_{0}=50 \mathrm{~W} \cdot\left(\mathrm{m}^{2} \cdot \mathrm{K}\right)^{-1}-$ heat flow by $x=L$;

$\alpha=0.1 \mathrm{dm} \cdot \mathrm{s}^{-1}-$ moisture transfer velocity.

We use the following parameters:

$$
s_{u}=q /\left(c_{p} \rho_{0}\right)=1.349, s_{T}=\sigma \cdot \mathcal{E} \cdot S /\left(c_{p} \rho_{0}\right)=3.2 \cdot 10^{-12}, f_{1}=F_{0} / k_{0}=333 .
$$

We have boundary conditions and initial conditions:

at $t=0$ and $x=0: T=20^{\circ} \mathrm{C}=293 \mathrm{~K}, u=25 \%$; at $x=L: k_{0} \frac{\partial T}{\partial x}=F_{0}, \frac{\partial u}{\partial x}=0$. 
For stability of the finite difference approximation the second equation of (9) is written in the following form:

$$
\partial u / \partial t=\partial\left(\left(e_{p} \partial u / \partial x\right)\right) / \partial x+\alpha \partial u / \partial x,
$$

where $\varepsilon_{p}=0.5 \cdot h \cdot \alpha$ - the small parameter, the factor of the artificial viscosity for approximation the derivative $\partial u / \partial x$ in uniform grid ( $h=0.01$ - grid step) with upwind difference.

When performing temperature calculations, we use Kelvin degrees, but the results of these calculations are given in degrees of Celsius.

The numerical experiment for the calculation of temperature and moisture concentrations in the wood-burning process was conducted with the Matlab programme "pdepe" and integral exponential type spline function (7), based on the experimental data obtained from testing wood samples for the European classification with the experimental equipment "SBI" at the Forest Faculty in The Forest and Wood Product Research and Development Institution of the Latvia University of Life Sciences and Technologies.

Fig. 9 and Fig. 10 illustrate the change in temperature and moisture concentration depending on the time at different places of the wood sample, i.e. at different $x$-coordinate values. The maximum of temperature $T_{\max }=835.16^{\circ} \mathrm{C}$ and the minimum of moisture content $u_{\min }=1.245 \%$ are reached at $x=5$.

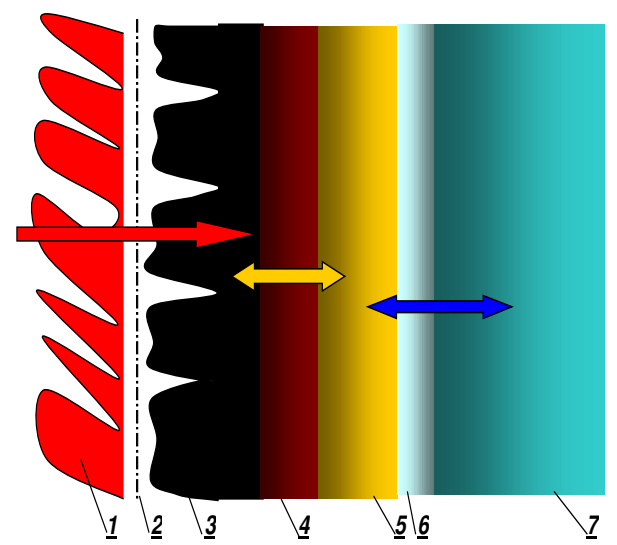

Fig. 7. Wood burning: 1 - flame; 2 - starting surface of the sample; 3 - charred wood;

4 - pyrolysis; 5 - dry wood; 6 - water evaporation; 7 - humid wood

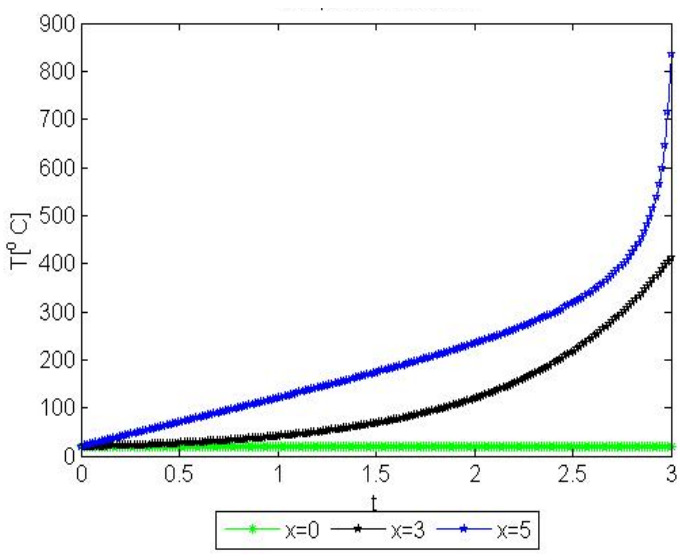

Fig. 9. Profile of temperature depending on $t$, $h$ at $x=0,3,5, T_{\max }=835.16^{\circ} \mathrm{C}$

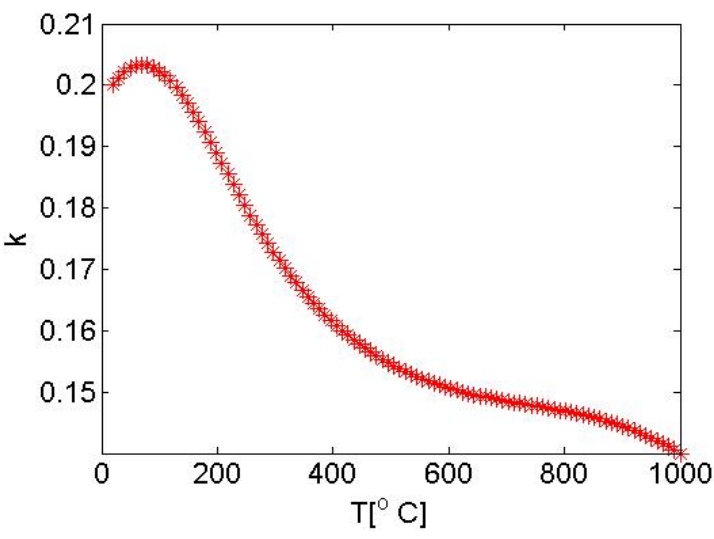

Fig. 8. Thermal conductivity depending on temperature $T$

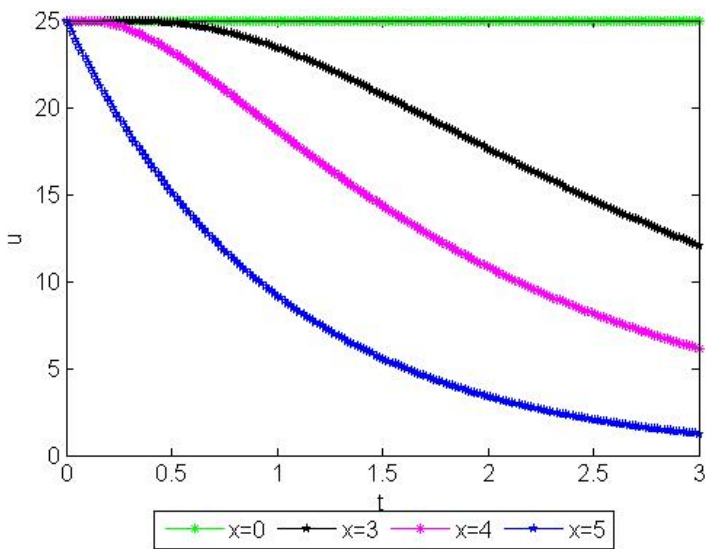

Fig. 10. Profile of moisture concentration depending on $t$, $\mathrm{h}$ at $x=0,3,4,5$, min $u=1.2445 \%$ 


\section{Conclusions}

1. On the basis of the results obtained in general terms (case of N-layers), it was proved that the exact solution of the studied boundary value problem (BVP), in a linear case, coincides with the corresponding approximated solution of the BVP, which in this case was the created exponential type spline function. This result is useful not only to solve the 1D boundary value problem (BVP) being studied, but also to solve the 3D and 2D BVP by reducing them to a $1 \mathrm{D}$ BVP with the conservative averaging method, as it allows to solve the acquired 1D BVP through the approximated solution (exponential or hyperbolic spline function) also where the exact solution of the 1D BVP cannot be obtained.

2. The nonstationary physical model for simple chemical reaction with temperature and 2 reactiondiffusion equations characterising the combustion process with Arrhenius kinetics is considered. The dependence of the combustion temperature on the axial velocity of fuel at the different values of the Levis number is studied. The influence of the molecular diffusivity and thermal conductivity on the temperature and reaction rate of the undisturbed flame flow is obtained.

3. When testing the combustion process with Arrhenius kinetics, based on numerical calculations, the following results were obtained.

- With increasing of the axial velocity $w$, there was observed the increasing of the maximal value of the temperature $T_{\max }$ for $L_{e}>1\left(L_{e}\right.$ - Lewis number) and the decreasing of $T_{\max }$ for $L_{e}<1$, but for $L_{e}=1-T_{\max }$ was not depended on $w$.

- Following an increase in the axial velocity $w$ at other constant parameters $\left(P_{1}, P_{2}, \alpha\right)$, a higher burning temperature $(T)$ was reached at the relevant points of the combustion chamber and at the fixed time values required for comparison. In addition, the experiment resulted in finding of the axial speed value $w$ at which the burning temperature remained constant throughout the entire phase of the combustion chamber, a very bright (well-visible) extension of the flame was visualised.

4. The spread of temperature and moisture in the wood sample in its burning process has been studied by solving the nonlinear system of the parabolic type of PDE being based on the experimental data obtained from testing wood samples at the Forest Faculty in The Forest and Wood Product Research and Development Institution of the Latvia University of Life Sciences and Technologies. The calculation required the use of thermal conductivity dependency on the temperature obtained by means of spline interpolation.

\section{References}

[1] Aboltins A., Kalis H., Kangro I. CAM with Special Splines for solving of Diffusion-Convection problems with Discontinuous Coefficients for Layered materials exposed to fire. Proceedings of International conference "18th International Scientific Conference ENGINEERING FOR RURAL DEVELOPMENT”, May 22-24, 2019, Jelgava, Latvia, vol. 18, 2019, pp. 1147-1154.

[2] Perre P., Keey R.B. 36 Drying of wood: principles and practices. Drying Technology. Taylor \& Francis Group, LLC, 2006, pp. 822-872.

[3] Salin J-G. Wood material Science and Engineering, vol.5, issue 2, 2010, pp. 900-985.

[4] Aboltins A. Kalis H., Pulkis K., Skujans J., Kangro I. On Mathematical Modelling of Heat Transfer problem for a two layered gypsum board products exposed to fire. Proceedings of International conference "16th International Scientific Conference ENGINEERING FOR RURAL DEVELOPMENT”, May 24-26, 2017, Jelgava, Latvia, vol. 16, 2017, pp.1369-1376.

[5] Aboltins A., Kalis H., Kangro I. On Mathematical Modelling of Heat and Moisture Distribution in the drying process for porous two layered gypsum board products. Proceedings of International conference "16th International Scientific Conference ENGINEERING FOR RURAL DEVELOPMENT”, May 24-26, 2017, Jelgava, Latvia, vol. 16, 2017, pp. 1369-1376.

[6] Powers J.M. Lecture notes on fundamentals of combustion. Univ. of Notre Dame, Indiana USA, 2012, pp. 5623-5637.

[7] Smooke M.D., Turnbull A.A., Mitchell R.E., Keyes D.E. Solution of two-dimensional axisymmetric laminar diffusion flames by adaptive boundary value methods. Proceedings of the NATO Advanced Research Workshop" Mathematical Modelling in Combustion and Related 
Topics”, April 27-30, 1987, Lyon, France, NATO ASI Series E: Applied Sciences, vol. 140, pp. 261-300.

[8] Choi J.J., Rusak Z., Kapila A.K. Numerical simulation of premixed chemical reactions with swirl. Combustion theory and modelling, vol. 6, No. 11, 2007, pp. 863-887.

[9] Kalis H., Marinaki M., Strautins U., Lietuvietis O. On the numerical simulation of the vortex breakdown in the combustion process with simple chemical reaction and axial magnetic field. Int. Journal of Differential Equations and Applications, vol. 14, No.3, 2015, pp. 325-350.

[10] Kalis H., Marinaki M., Strautins U., Lietuvietis O. On the numerical simulation of the combustion process with simple chemical reaction, Proceedings of the 7-th Baltic Heat Transfer conference "Advances in Heat Transfer", August 24-26, 2015, pp. 175-180.

[11]Buikis A., Kalis H., Kangro I. Special Splines of Exponential Type for the Solutions of Mass Transfer Problems in Multilayer Domains. Mathematical Modelling and Analysis, vol. 21, issue 4, 2016, pp. 450-465.

[12] Kalis H., Barmina I., Zake M., Koliskins A. Mathematical modelling and experimental study of electrodynamic control of swirling flame flows. Proceedings of International conference"15th International Scientific Conference ENGINEERING FOR RURAL DEVELOPMENT", May 2527, 2016, Jelgava, Latvia, vol. 15, 2016, pp. 54-59.

[13] Syred N., Beer J.M. Combustion in Swirling Flows: A review Combustion and Flame 23, 1974, pp. 143-201.

[14] Mujumdar A.S. Hanbook of industrial drying. 3. Edition. Taylor \& Francis, 2007. 320 p.

[15] Atreya A. The science of wood combustion. Proc. Indian Acad. Sci., vol. 5, Pt 4, 1982, pp. 259268. 